\title{
EIGENVALUE STABILITY FOR MULTIVALUED OPERATORS
}

\author{
Philippe Lavilledieu — Alberto Seeger
}

Dedicated to the memory of Juliusz P. Schauder

\begin{abstract}
Let $\sigma(F)$ denote the point spectrum of a multivalued operator $F: H \rightrightarrows H$ defined over a real Hilbert space. The aim of this note is to explore the continuity properties of the spectral mapping $F \rightrightarrows \sigma(F)$
\end{abstract}

\section{Introduction}

In the sequel $(H,\langle\cdot, \cdot\rangle)$ is a real Hilbert space, and $P(H)$ stands for the collection of all subsets of $H$. We follow the standard practice which consists in identifying a function $F: H \rightarrow P(H)$ with the multivalued operator $F: H \rightrightarrows$ $H$. The double-arrow notation has the advantage of stressing the multivalued character of $F$. Let

$$
\sigma(F):=\{\lambda \in \mathbb{R}: \lambda \text { is an eigenvalue of } F\}
$$

denote the point spectrum of $F: H \rightrightarrows H$. Recall that a real number $\lambda$ is said to be an eigenvalue of $F$ if there is a vector $x \in H \backslash\{0\}$ verifying $\lambda x \in F(x)$. Such $x$ is called an eigenvector of $F$. For a brief historic account on the eigenvalue analysis of multivalued systems, the reader can consult Seeger $(1998,1999)$ and the references therein.

2000 Mathematics Subject Classification. 47H04, 47H12, 58C40.

Key words and phrases. Multivalued operator, eigenvalue, point spectrum, Painlevé-Kuratowski convergence, homogenization, pseudo-similarity transformation.

This paper was written in part during the second author's stay at the Hanoi Institute of Mathematics, Vietnam. Thanks are due to this institution for hospitality and working facilities. 
The purpose of this note is to explore the continuity properties of the spectral mapping $F \rightrightarrows \sigma(F)$. Roughly speaking, we would like to know how does the set $\sigma(F)$ behave when $F$ is subject to small perturbations. From a qualitative point of view, the question under consideration is the following one: does the implication

$$
F=\lim F_{k} \Rightarrow \sigma(F)=\lim \sigma\left(F_{k}\right)
$$

hold for appropriate and reasonable convergence notions?

Observe that the above implication expresses (sequential) continuity of the mapping $\sigma$ at the reference argument $F$. The continuity analysis of $\sigma$ has been traditionally carried out in the context of the Banach algebra of bounded linear operators. In such a setting, a wealth of interesting results have been obtained since the pioneering work by Newburgh (1951). For relevant bibliographic information, the reader can consult Conway and Morrel (1979), and Halmos (1982).

Our treatment of the subject is not inspired by the classical sources, and this is because we are dealing now with multivalued operators. We leave aside the beautiful and powerful spectral theory for bounded linear operators, and adjust somehow our mathematical tools in order to cope with difficulties arising from multivaluedness.

The convergence of sets and multivalued operators can be understood in manifold ways. To keep the lenght of our exposition at bay, only the case of Painlevé-Kuratowski convergence will be discussed here.

Definition 1.1 (convergence of sets). Let $\left\{C_{k}\right\}_{k \in \mathbb{N}}$ be a sequence of sets in a topological space $Z$. The outer-limit of $\left\{C_{k}\right\}_{k \in \mathbb{N}}$ is defined by

$$
z \in \text { outlim } C_{k} \Leftrightarrow\left\{\begin{array}{l}
\text { there are a sequence }\left\{z^{k}\right\}_{k \in \mathbb{N}} \rightarrow z \\
\text { and a strictly increasing function } \varphi: \mathbb{N} \rightarrow \mathbb{N} \\
\text { such that } z^{k} \in C_{\varphi(k)} \text { for all } k \in \mathbb{N} .
\end{array}\right.
$$

The inner-limit of $\left\{C_{k}\right\}_{k \in \mathbb{N}}$ corresponds to the set given by

$$
z \in \operatorname{innlim} C_{k} \Leftrightarrow\left\{\begin{array}{l}
\text { there are a sequence }\left\{z^{k}\right\}_{k \in \mathbb{N}} \rightarrow z \text { and an integer } \\
k_{0} \in \mathbb{N} \text { such that } z^{k} \in C_{k} \text { for all } k \geq k_{0} .
\end{array}\right.
$$

If outlim $C_{k}$ and $\operatorname{innlim} C_{k}$ coincide, then the common set, denoted by $\lim C_{k}$, is referred to as the Painlevé-Kuratowski limit of $\left\{C_{k}\right\}_{k \in \mathbb{N}}$. The empty set is not excluded as a possible Painlevé-Kuratowski limit.

The theory of set convergence provides a way of approximating multivalued operators through convergence of graphs. In what follows, $M(H)$ denotes the class of multivalued operators from $H$ into $H$. As usual,

$$
\operatorname{Gr} F:=\{(x, y) \in H \times H: y \in F(x)\}
$$

stands for the graph of $F: H \rightrightarrows H$. 
DEFINITION 1.2 (convergence of multivalued operators). Let $\left\{F_{k}\right\}_{k \in \mathbb{N}}$ be a sequence in $M(H)$. The multivalued operators outlim $F_{k}: H \rightrightarrows H$ and innlim $F_{k}: H \rightrightarrows H$ are defined respectively by

$$
\begin{aligned}
& \operatorname{Gr}\left[\operatorname{outlim} F_{k}\right]=\operatorname{outlim}\left[\operatorname{Gr} F_{k}\right], \\
& \operatorname{Gr}\left[\operatorname{innlim} F_{k}\right]=\operatorname{innlim}\left[\operatorname{Gr} F_{k}\right] .
\end{aligned}
$$

The notation $F=\lim F_{k}$ indicates that $\left\{\operatorname{Gr} F_{k}\right\}_{k \in \mathbb{N}}$ converges in the Painlevé Kuratowski sense toward Gr F.

This note aims at determining the continuity region of $\sigma$. As a first step in this direction, we single out some classes of multivalued operators $F_{k}: H \rightrightarrows H$ for which one can write a formula ofthe type

$$
\sigma\left(\lim F_{k}\right)=\lim \sigma\left(F_{k}\right)
$$

Such an equality can be derived by combining two inclusions, namely

$$
\begin{aligned}
\operatorname{outlim} \sigma\left(F_{k}\right) \subset \sigma\left(\operatorname{outlim} F_{k}\right) & \text { (outer-semicontinuity), } \\
\sigma\left(\operatorname{innlim} F_{k}\right) \subset \operatorname{innlim} \sigma\left(F_{k}\right) & \text { (inner-semicontinuity). }
\end{aligned}
$$

A quick examination at simple examples indicates that $\sigma$ is unlikely to behave in a continuous manner, but it may enjoy at least one of the two types of semicontinuity.

EXAMPLE 1.3 (lack of outer-semicontinuity). Let the multivalued operator $F_{k}: H \rightrightarrows H$ be defined by $F_{k}(x)=\{y \in H:\|y-x\| \leq 1 / k\}$. The sequence $\left\{F_{k}\right\}_{k \in \mathbb{N}}$ converges in the Painlevé-Kuratowski sense toward the singlevalued operator $x \in H \mapsto F(x)=\{x\}$. A direct computation shows that $\sigma\left(F_{k}\right)=\mathbb{R}$ for all $k \in \mathbb{N}$. As a consequence, $\sigma(F)=\{1\}$ is strictly contained in $\lim \sigma\left(F_{k}\right)=\mathbb{R}$.

EXAMPLE 1.4 (lack of inner-semicontinuity). Consider the linear operator $F_{k}: \mathbb{R}^{2} \rightarrow \mathbb{R}^{2}$ given by

$$
F_{k}(x)=A_{k} x, \text { with } A_{k}=\left[\begin{array}{cc}
0 & \frac{1}{k} \\
-\frac{1}{k} & 0
\end{array}\right] .
$$

The Painlevé-Kuratowski limit of $\left\{F_{k}\right\}_{k \in \mathbb{N}}$ exists and is given by $F(x)=0$ for all $x \in \mathbb{R}^{2}$. Since $\sigma\left(F_{k}\right)=\emptyset$ for all $k \in \mathbb{N}$, it follows that $\lim \sigma\left(F_{k}\right)=\emptyset$ is strictly contained in $\sigma(F)=\{0\}$.

\section{Preliminary outer-semicontinuity results}

As mentioned before, a real number $\lambda$ is an eigenvalue of $F: H \rightrightarrows H$ if the eigenset

$$
E(\lambda, F):=\{x \in H: \lambda x \in F(x)\}
$$


contains a nonzero vector. As a matter of independent interest, we shall discuss briefly the continuity properties of the mapping $(\lambda, F) \rightrightarrows E(\lambda, F)$.

For notational convenience, one writes

$$
E(\Lambda, F):=\bigcup_{\lambda \in \Lambda} E(\lambda, F) \quad \text { for any } \Lambda \subset \mathbb{R} .
$$

As a particular case of Definition 1.1, one sees that outlim $\lambda_{k}:=\operatorname{outlim}\left\{\lambda_{k}\right\}$ corresponds to the set of all cluster points of the sequence $\left\{\lambda_{k}\right\}_{k \in \mathbb{N}}$.

Proposition 2.1 (outer-semicontinuity of eigensets). Consider a bounded sequence $\left\{\lambda_{k}\right\}_{k \in \mathbb{N}} \subset \mathbb{R}$. Let $\Lambda \subset \mathbb{R}$ be a set containing outlim $\lambda_{k}$, and $F: H \rightrightarrows H$ be a multivalued operator such that $\operatorname{Gr}\left[\operatorname{outlim} F_{k}\right] \subset \mathrm{Gr} F$. Then

$$
\operatorname{outlim} E\left(\lambda_{k}, F_{k}\right) \subset E(\Lambda, F) .
$$

Proof. Let $x \in \operatorname{outlim} E\left(\lambda_{k}, F_{k}\right)$. For some strictly increasing $\varphi: \mathbb{N} \rightarrow \mathbb{N}$ and some $\left\{x^{k}\right\}_{k \in \mathbb{N}} \rightarrow x$, one has

$$
x^{k} \in E\left(\lambda_{\varphi(k)}, F_{\varphi(k)}\right) \quad \text { for all } k \in \mathbb{N} .
$$

The above condition can be written also in the form

$$
\left(x^{k}, \lambda_{\varphi(k)} x^{k}\right) \in \operatorname{Gr} F_{\varphi(k)} \quad \text { for all } k \in \mathbb{N} .
$$

Take now an increasing function $\psi: \mathbb{N} \rightarrow \mathbb{N}$ so that $\left\{\lambda_{\varphi(\psi(k))}\right\}_{k \in \mathbb{N}}$ converges to some $\lambda \in \mathbb{R}$. From the relation

$$
\left(x^{\psi(k)}, \lambda_{\varphi(\psi(k))} x^{\psi(k)}\right) \in \operatorname{Gr} F_{\varphi(\psi(k))} \quad \text { for all } k \in \mathbb{N},
$$

it follows that

$$
(x, \lambda x) \in \text { outlim Gr } F_{k}=\operatorname{Gr}\left[\text { outlim } F_{k}\right] \subset \operatorname{Gr} F .
$$

Hence, $x \in E(\lambda, F)$. Since $\lambda \in$ outlim $\lambda_{k} \subset \Lambda$, one gets the desired conclusion $x \in E(\Lambda, F)$.

Corollary 2.2. Suppose that $\left\{\lambda_{k}\right\}_{k \in \mathbb{N}}$ converge to $\lambda \in \mathbb{R}$. Let $F: H \rightrightarrows H$ be a multivalued operator such that $\operatorname{Gr}\left[\operatorname{outlim} F_{k}\right] \subset \mathrm{Gr} F$. Then

$$
\operatorname{outlim} E\left(\lambda_{k}, F_{k}\right) \subset E(\lambda, F) .
$$

Proof. It suffices to choose $\Lambda=\{\lambda\}$.

Now we establish our first outer-semicontinuity result for the spectral mapping $\sigma$. This result is not interesting by itself, but it will have several useful consequences. We state it bellow as a lemma. Recall that the notation

$$
D(F):=\{x \in H: F(x) \neq \emptyset\}
$$

refers to the domain of $F: H \rightrightarrows H$. 
Lemma 2.3. Consider a sequence $\left\{F_{k}\right\}_{k \in \mathbb{N}}$ of multivalued operators $F_{k}$ : $H \rightrightarrows H$ such that:

(a) for some $k_{0} \in \mathbb{N}$, the set $\bigcup_{k \geq k_{0}} D\left(F_{k}\right)$ is relatively compact in $H$,

(b) $0 \notin\left(\right.$ outlim $\left.F_{k}\right)(0)$.

Then, outlim $\sigma\left(F_{k}\right) \subset \sigma\left(\right.$ outlim $\left.F_{k}\right)$.

Proof. Take any $\lambda$ in outlim $\sigma\left(F_{k}\right)$. This means that for some strictly increasing funtion $\varphi: \mathbb{N} \rightarrow \mathbb{N}$, and some $\left\{\lambda_{k}\right\}_{k \in \mathbb{N}} \rightarrow \lambda$, it is possible to write

$$
\lambda_{k} \in \sigma\left(F_{\varphi(k)}\right) .
$$

Take $x^{k} \in H \backslash\{0\}$ so that

$$
\lambda_{k} x^{k} \in F_{\varphi(k)}\left(x^{k}\right) \text { for all } k \in \mathbb{N} .
$$

Due to assumption (a), the sequence $\left\{x^{k}\right\}_{k \in \mathbb{N}}$ lies in some compact set of $H$. Take a strictly increasing function $\psi: \mathbb{N} \rightarrow \mathbb{N}$ so that $\left\{x^{\psi(k)}\right\}_{k \in \mathbb{N}}$ is convergent, say to $x \in X$. From the relation

$$
\left(x^{\psi(k)}, \lambda_{\psi(k)} x^{\psi(k)}\right) \in \operatorname{Gr} F_{\varphi(\psi(k))} \quad \text { for all } k \in \mathbb{N},
$$

it follows that

$$
(x, \lambda x) \in \operatorname{Gr}\left[\operatorname{outlim} F_{k}\right] .
$$

Assumption (b) guarantees that $x$ is a nonzero vector, showing in this way that $\lambda \in \sigma\left(\operatorname{outlim} F_{k}\right)$.

The interpretation of assumption (a) in Lemma 2.3 is straightforward: there should be a compact set $M \subset H$ such that

$$
D\left(F_{k}\right) \subset M \text { for all } k \in \mathbb{N} \text { sufficiently large. }
$$

For the sake of brevity, one says that the collection $\left\{D\left(F_{k}\right)\right\}_{k \in \mathbb{N}}$ is uniformly compact. This very stringent requirement leaves aside several important classes of multivalued operators. Assumption (b) in Lemma 2.3 is also quite strong: for instance, it rules out the usual case in which $0 \in F_{k}(0)$ for all $k \in \mathbb{N}$. Fortunately, it is possible to extend Lemma 2.3 in such a way as to circumvect these severe constraints.

\section{Outer-semicontinuity via homogenization}

Our first extension method is heavily influenced by the following key observation: the point spectrum of a multivalued operator is invariant under homogenization. Thus, one could consider

$$
F_{k}=\text { homogenized version of } S_{k},
$$

with $\left\{S_{k}\right\}_{k \in \mathbb{N}}$ satisfying the assumptions stated in Lemma 2.3. In the theorem below, we write down more precisely what we have in mind. We state first: 
Lemma 3.1. Let $S: H \rightrightarrows H$ be a multivalued operator. For each $T \subset \mathbb{R}$, let $T \bullet S: H \rightrightarrows H$ be defined by

$$
\operatorname{Gr}[T \bullet S]=T \operatorname{Gr} S=\{t(x, y): t \in T,(x, y) \in \operatorname{Gr} S\} .
$$

Then, $\sigma(T \bullet S)=\sigma(S)$, provided $T$ intersects $\mathbb{R} \backslash\{0\}$.

Proof. It is immediate.

Theorem 3.2 (outer-semicontinuity via homogenization). Let $\left\{S_{k}\right\}_{k \in \mathbb{N}}$ be a sequence of multivalued operators $S_{k}: H \rightrightarrows H$ such that:

(a) for some $k_{0} \in \mathbb{N}$, the set $\bigcup_{k \geq k_{0}} D\left(S_{k}\right)$ is relatively compact in $H$,

(b) $0 \notin\left[\right.$ outlim $\left.S_{k}\right](0)$.

Let $\left\{F_{k}\right\}_{k \in \mathbb{N}}$ be given by the relation

$$
F_{k}:=T_{k} \bullet S_{k} \quad \text { for all } k \in \mathbb{N},
$$

where each $T_{k} \subset \mathbb{R}$ is assumed to contain a common nonzero real number (i.e., $\bigcap_{k \in \mathbb{N}} T_{k}$ intersects $\left.\mathbb{R} \backslash\{0\}\right)$. Then,

$$
\operatorname{outlim} \sigma\left(F_{k}\right) \subset \sigma\left(\operatorname{outlim} F_{k}\right) \text {. }
$$

Proof. Lemma 3.1 yields the equality $\sigma\left(F_{k}\right)=\sigma\left(S_{k}\right)$. Hence

$$
\operatorname{outlim} \sigma\left(F_{k}\right)=\operatorname{outlim} \sigma\left(S_{k}\right) \subset \sigma\left(\operatorname{outlim} S_{k}\right),
$$

the latter inclusion being a consequence of Lemma 2.3. Pick up any $a \neq 0$ in the intersection of the $T_{k}^{\prime} s$. If $a=1$, then we are done. Indeed, the assumption $1 \in T_{k}$ guarantees the inclusion $\operatorname{Gr} S_{k} \subset \operatorname{Gr} F_{k}$, from where one obtains $\sigma\left(\right.$ outlim $\left.S_{k}\right) \subset$ $\sigma\left(\operatorname{outlim} F_{k}\right)$. If $a \neq 1$, then one writes

$$
T_{k} \bullet S_{k}=\widetilde{T}_{k} \bullet \widetilde{S}_{k}
$$

with

$$
\widetilde{T}_{k}=\left\{t / a: t \in T_{k}\right\} \quad \text { and } \quad \operatorname{Gr} \widetilde{S}_{k}=\left\{a(x, y):(x, y) \in \operatorname{Gr} S_{k}\right\} .
$$

It suffices then to apply the previous argument to the new decomposition $F_{k}=$ $\widetilde{T}_{k} \bullet \widetilde{S}_{k}$. Observe that each $\widetilde{T}_{k}$ contains the element 1 , and the sequence $\left\{\widetilde{S}_{k}\right\}_{k \in \mathbb{N}}$ inherits the assumptions made on $\left\{S_{k}\right\}_{k \in \mathbb{N}}$. This completes the proof.

Recall that a multivalued operator $F: H \rightrightarrows H$ is declared positively homogeneous if

$$
F(t x)=t F(x) \text { for all } x \in H \text {, and all } t>0 .
$$

This property amounts to saying that Gr $F$ is a cone in the sense that $t \operatorname{Gr} F \subset$ Gr $F$ for all $t>0$. Examples of positively homogeneous multivalued operators abound in the literature. Besides linear relations in the sense of Arens (1961), 
they include convex processes (Rockafellar, 1970), processes defined by linear complementarity conditions (Seeger, 1999), and fans (Ioffe, 1981). In general,

$F: H \rightrightarrows H$ is positively homogeneous $\Leftrightarrow\left\{\begin{array}{l}\text { there exist a cone } T \subset \mathbb{R} \\ \text { and an operator } S: H \rightrightarrows H \\ \text { such that } F=T \bullet S .\end{array}\right.$

Corollary 3.3. Let the real Hilbert space $(H,\langle\cdot, \cdot\rangle)$ be finite dimensional. If $\left\{F_{k}\right\}_{k \in \mathbb{N}}$ is a sequence of positively homogeneous operators $F_{k}: H \rightrightarrows H$, then

$$
\operatorname{outlim} \sigma\left(F_{k}\right) \subset \sigma\left(\operatorname{outlim} F_{k}\right) \text {. }
$$

Proof. For each $k \in \mathbb{N}$, define $S_{k}: H \rightrightarrows H$ by the relation

$$
\operatorname{Gr} S_{k}=\operatorname{Gr} F_{k} \cap \Sigma^{H \times H},
$$

where

$$
\Sigma^{H \times H}:=\{(x, y) \in H \times H:\langle x, x\rangle+\langle y, y\rangle=1\}
$$

stands for the unit sphere of the product space $H \times H$. Due to the positive homogeneity of $F_{k}$, one knows that $\operatorname{Gr} F_{k}$ is a cone in $H \times H$. There is no loss of generality in assuming that $(0,0) \in \operatorname{Gr} F_{k}$, so one can write

$$
\operatorname{Gr} F_{k}=\mathbb{R}_{+} \operatorname{Gr} S_{k} .
$$

Thus $F_{k}=\mathbb{R}_{+} \bullet S_{k}$, with $\left\{S_{k}\right\}_{k \in \mathbb{N}}$ being a sequence of multivalued operators as in Theorem 2.5. Observe that each $D\left(S_{k}\right)$ is contained in the compact set $\Sigma^{H}:=\{x \in H:\langle x, x\rangle=1\}$. The condition $0 \notin\left(\operatorname{outlim} S_{k}\right)(0)$ is implied by the inclusion $\operatorname{Gr}\left[\right.$ outlim $\left.S_{k}\right] \subset \Sigma^{H \times H}$.

The above corollary does not extend to infinite dimensional Hilbert spaces, even if the $F_{k}^{\prime} s$ are linear operators.

EXAMPLE 3.4 (failure of outer-semicontinuity in infinite dimensional spaces). Consider the Hilbert space $H=L_{2}[0,1]$ equipped with the usual product

$$
\langle u, v\rangle:=\int_{0}^{1} u(s) v(s) d s .
$$

Let the sequence $\left\{u_{k}\right\}_{k \in \mathbb{N}} \subset H$ be defined by

$$
u_{k}(s)= \begin{cases}\sqrt{k(k+1)} & \text { if } s \in\left[\frac{1}{k}-\frac{1}{2 k(k+1)}, \frac{1}{k}+\frac{1}{2 k(k+1)}\right], \\ 0 & \text { otherwise. }\end{cases}
$$

This sequence lies in the unit sphere of $H$, but is does not admit a converging subsequence. Indeed, the equality

$$
\left\|u_{k}-u_{\ell}\right\|=\left\{\int_{0}^{1}\left[u_{k}(s)-u_{\ell}(s)\right]^{2} d s\right\}^{1 / 2}=2 \quad \text { for all } k, \ell \in \mathbb{N}, k \neq \ell,
$$


shows that none of the subsequences of $\left\{u_{k}\right\}_{k \in \mathbb{N}}$ is a Cauchy sequence. Consider now the linear operators $F_{k}: H \rightarrow H$ given by

$$
F_{k}(x)=\left\langle a_{k}, x\right\rangle u_{k} \quad \text { for all } x \in H,
$$

with $\left\{a_{k}\right\}_{k \in \mathbb{N}} \subset H$ being constructed as follows:

$$
a_{2 k}=(-1)^{k} u_{2 k}, \quad a_{2 k+1}=\frac{1}{2 k+1} u_{2 k+1} .
$$

A little bit of calculation yields

$$
\left(\operatorname{outlim} F_{k}\right)(x)=0 \quad \text { for all } x \in H, \quad \sigma\left(F_{k}\right)=\left\{\left\langle a_{k}, u_{k}\right\rangle\right\},
$$

from where one deduces that outlim $\sigma\left(F_{k}\right)=\{-1,0,1\}$ is not contained in $\sigma\left(\right.$ outlim $\left.F_{k}\right)=\{0\}$.

\section{Outer-semicontinuity via pseudo-similarity}

The second way of extending Lemma 2.3 is by using pseudo-similarity transformations. This new terminology must be understood in the sense indicated below:

Definition 4.1. A singlevalued operator $Q: H \rightarrow H$ is said to be a pseudosimilarity transformation if the following three conditions are fulfilled:

(a) $Q$ is not the null mapping, i.e. $Q(x) \neq 0$ for some $x \in H$;

(b) if $\left\{Q\left(x^{k}\right)\right\}_{k \in \mathbb{N}}$ converges to a nonzero vector, then $\left\{x^{k}\right\}_{k \in \mathbb{N}}$ has a nonzero cluster point

(c) there is a locally bounded function $\xi: \mathbb{R} \rightarrow \mathbb{R}$ such that

$$
Q(t x)=\xi(t) Q(x) \text { for all } x \in H, \text { for all } t \in \mathbb{R} .
$$

Such $\xi$ is referred to as the scaling function associated with $Q$.

Some comments on the above definition are in order. First of all, the condition (b) in Definition 4.1 yields

$$
x=0 \Rightarrow Q(x)=0,
$$

but the converse implication is not necessarily true. Just think of the pseudosimilarity transformation $Q: \mathbb{R}^{2} \rightarrow \mathbb{R}^{2}$ given by

$$
Q(x)= \begin{cases}0 & \text { if } x_{1} x_{2}=0 \\ x & \text { if } x_{1} x_{2} \neq 0\end{cases}
$$

Secondly, local boundedness of $\xi: \mathbb{R} \rightarrow \mathbb{R}$ means that for all $t \in \mathbb{R}$ there is a neighbourhood $V$ of $t$ such that $\xi(V)$ is bounded.

Of course, local boundedness is a property that is in general weaker than continuity. The next proposition, however, shows that scaling functions are necessarily continuous. In fact, scaling functions have a very particular form: 
Proposition 4.2 (characterization of scaling functions). Let $\xi: \mathbb{R} \rightarrow \mathbb{R}$ be the scaling function of some pseudo-similarity transformation. Then,

(a) $\xi$ is even or odd,

(b) there is a positive real number $p$ such that $\xi(t)=t^{p}$ for all $t \geq 0$.

Proof. Definition 4.1 yields the following functional equation

$$
\left\{\begin{array}{l}
\xi(t s)=\xi(t) \xi(s) \quad \text { for } t, s \in \mathbb{R}, \\
\lim _{t \rightarrow \infty} \xi(t)=\infty
\end{array}\right.
$$

from where one obtains the desired conclusion.

If $Q: H \rightarrow H$ is a continuous linear bijection, then $Q$ is a pseudo-similarity transformation with scaling function $t \in \mathbb{R} \mapsto \xi(t)=t$. More generally:

Proposition 4.3. If $Q: H \rightarrow H$ is a continuous linear injection with closed range, then $Q$ is a pseudo-similarity transformation with scaling function $t \in \mathbb{R} \mapsto \xi(t)=t$.

Proof. Checking the property (b) in Definition 4.1 is the only nontrivial part of the proof. Suppose that $\left\{Q\left(x^{k}\right)\right\}_{k \in \mathbb{N}}$ converges to a nonzero vector $y \in H$. Since each $Q\left(x^{k}\right)$ lies in the closed set $\operatorname{Im} Q=\{Q x: x \in H\}$, so does $y$. The assumptions made on $Q$ guarantee the existence of a continuous linear bijection $L: \operatorname{Im} Q \rightarrow H$ such that $L \circ Q: H \rightarrow H$ is the identity mapping of $H$ (one says that $L$ is a left-inverse of $Q)$. It follows that $L\left(Q\left(x^{k}\right)\right)=x^{k}$ converges to the nonzero vector $L y$.

An interesting example of nonlinear pseudo-similarity transformation is given below.

ExAmPle 4.4. Let $H=S_{n}$ be the space of symmetric matrices of order $n \times n$. To evaluate $Q: H \rightarrow H$ at a given $A \in S_{n}$, one first obtains the polar decomposition

$$
A=\sum_{i=1}^{n} \lambda_{i} u_{i} u_{i}^{T}
$$

and then one sets

$$
Q(A):=\sum_{i=1}^{n} \gamma\left(\lambda_{i}\right) u_{i} u_{i}^{T} .
$$

The $\lambda_{i}^{\prime} s$ are the eigenvalues of $A$, and the $u_{i}^{\prime} s$ are the corresponding normalized eigenvectors. It can be shown that $Q$ is a pseudo-similarity transformation if $\gamma: \mathbb{R} \rightarrow \mathbb{R}$ has any of the following forms:

$$
\begin{array}{ll}
\gamma(t)=|t|^{p} & \text { with } p \in] 0, \infty[ \\
\gamma(t)=t^{p} & \text { with } p \in\{1,2,3, \ldots\}, \\
\gamma(t)=\sqrt[p]{t} & \text { with } p \in\{1,3,5, \ldots\}
\end{array}
$$


Proposition 4.5. Let $Q_{1}$ and $Q_{2}$ be pseudo-similarity transformations with scaling functions $\xi_{1}$ and $\xi_{2}$, respectively. Suppose that $Q_{1} \circ Q_{2}$ is not the null mapping. Then $Q_{1} \circ Q_{2}$ is a pseudo-similarity transformation with scaling function $\xi_{1} \circ \xi_{2}$.

Proof. Just apply Definition 4.1.

Definition 4.6. One says that $F: H \rightrightarrows H$ is pseudo-similar to $S: H \rightrightarrows H$ if there is a pseudo-similar transformation $Q: H \rightarrow H$ such that

$$
F=Q^{-} \circ S \circ Q,
$$

where $Q^{-}: H \rightrightarrows H$ is defined by $Q^{-}(v):=\{u \in H: Q(u)=v\}$.

The symbol "o" in Definition 4.6 refers to the composition operation for multivalued operators. As a matter of computation, one obtains

$$
\begin{aligned}
y \in\left[Q^{-} \circ S \circ Q\right](x) & \Leftrightarrow y \in \bigcup\left\{Q^{-}(v): v \in S(Q(x))\right\} \\
& \Leftrightarrow \text { there is } v \in S(Q(x)) \text { such that } y \in Q^{-}(v) \\
& \Leftrightarrow Q(y) \in S(Q(x)) .
\end{aligned}
$$

In other words,

$$
\operatorname{Gr}\left[Q^{-} \circ S \circ Q\right]=\{(x, y) \in H \times H:(Q(x), Q(y)) \in \operatorname{Gr} S\} .
$$

TheOREM 4.7 (outer-semicontinuity via pseudo-similarity). Let $\left\{S_{k}\right\}_{k \in \mathbb{N}}$ be a sequence of multivalued operators $S_{k}: H \rightrightarrows H$ as in Theorem 3.2. Let $\left\{F_{k}\right\}_{k \in \mathbb{N}}$ be given by the relation

$$
F_{k}:=Q^{-} \circ S_{k} \circ Q \text { for all } k \in \mathbb{N},
$$

where $Q: H \rightarrow H$ is a pseudo-similarity transformation. Then,

$$
\operatorname{outlim} \sigma\left(F_{k}\right) \subset \sigma\left(\operatorname{outlim} F_{k}\right) \text {. }
$$

Proof. Let $\lambda \in \operatorname{outlim} \sigma\left(F_{k}\right)$. As in theproof of Lemma 2.3, one shows that

$$
\left(x^{k}, \lambda_{k} x^{k}\right) \in \operatorname{Gr} F_{\varphi(k)} \quad \text { for all } k \in \mathbb{N},
$$

for some strictly increasing $\varphi: \mathbb{N} \rightarrow \mathbb{N}$, and for suitable sequences $\left\{x^{k}\right\} \subset H \backslash\{0\}$ and $\left\{\lambda_{k}\right\}_{k \in \mathbb{N}} \rightarrow \lambda$. The very definition of $\left\{F_{k}\right\}_{k \in \mathbb{N}}$ yields

$$
\left(Q\left(x^{k}\right), Q\left(\lambda_{k} x^{k}\right)\right) \in \operatorname{Gr} S_{\varphi(k)} \quad \text { for all } k \in \mathbb{N},
$$

from where one obtains

$$
\left(Q\left(x^{k}\right), \xi\left(\lambda_{k}\right) Q\left(x^{k}\right)\right) \in \operatorname{Gr} S_{\varphi(k)} \quad \text { for all } k \in \mathbb{N} .
$$

Observe that $\left\{Q\left(x^{k}\right)\right\}_{k \in \mathbb{N}}$ lies in some compact set of $H$, and $\left\{\xi\left(\lambda_{k}\right)\right\}_{k \in \mathbb{N}}$ is bounded. Consequently, there is a strictly increasing $\psi: \mathbb{N} \rightarrow \mathbb{N}$ such that 
$\left\{Q\left(x^{\psi(k)}\right)\right\}_{k \in \mathbb{N}}$ converges to some $y \in H$, and $\left\{\xi\left(\lambda_{\psi(k)}\right)\right\}_{k \in \mathbb{N}}$ converges to some $\mu \in \mathbb{R}$. One has necessarily

$$
(y, \mu y) \in \operatorname{Gr}\left[\operatorname{outlim} S_{k}\right],
$$

which shows that $y$ is a nonzero vector. Since $Q$ is a pseudo-similarity transformation, it follows that $\left\{x^{\psi(k)}\right\}_{k \in \mathbb{N}}$ admits a subsequence converging to some $x \in H \backslash\{0\}$. The conclusion is that

$$
(x, \lambda x) \in \operatorname{Gr}\left[\operatorname{outlim} F_{k}\right] \text {, with } x \neq 0 .
$$

In other words, $\lambda \in \sigma\left(\operatorname{outlim} F_{k}\right)$.

Observe that the collection $\left\{D\left(S_{k}\right)\right\}_{k \in \mathbb{N}}$ is uniformly compact, but it may well happen that $\left\{D\left(F_{k}\right)\right\}_{k \in \mathbb{N}}$ does not enjoy this property. In other words, uniform compactness is not preserved by pseudo-similarity transformations. Thus, Theorem 4.7 is a genuine generalization of Lemma 2.3 .

\section{Inner-semicontinuity results}

As far as the Banach algebra of bounded linear operators is concerned, it is well known that inner-semicontinuity of the spectral mapping is something quite difficult to achieve. There is no reason to expect a better innersemicontinuity behavior in the multivalued case. Attempts at ascertaining the inclusion $\sigma\left(\operatorname{innlim} F_{k}\right) \subset \operatorname{innlim} \sigma\left(F_{k}\right)$ will be successful only under very stringent assumptions on the sequence $\left\{F_{k}\right\}_{k \in \mathbb{N}}$.

To prepare the ground for establishing our next theorem, a brief review of the concept of numerical range will be helpful. Recall that the numerical range (or field of values) of a linear operator $A: H \rightarrow H$ is defined by

$$
W(A):=\{\langle x, A x\rangle:\langle x, x\rangle=1\},
$$

i.e. it is the set of values assumed by the quadratic form $\langle x, A x\rangle$ when $x$ ranges over the unit sphere $\sum_{H}$. The equivalent characterization

$$
W(A)=\left\{\frac{\langle x, A x\rangle}{\langle x, x\rangle}: x \neq 0\right\}
$$

can be used as starting point for defining the numerical range of a multivalued operator.

Definition 5.1. The numerical range of $F: H \rightrightarrows H$ is the subset of $\mathbb{R}$ given by

$$
W(F):=\left\{\frac{\langle x, y\rangle}{\langle x, x\rangle}: x \neq 0,(x, y) \in \operatorname{Gr} F\right\} .
$$

Some basic properties of the numerical range mapping $W$ are recorded in the next proposition. These properties follow rather easily from Definition 5.1, so they are given without proof. 
Proposition 5.2. The numerical range mapping $W: M(H) \rightrightarrows R$ enjoys the following properties:

(a) (homogeneity) $W(t F)=t W(F)$ for all $t \in \mathbb{R}$, for all $F \in M(H)$, with $t F \in M(H)$ being defined by $(t F)(x):=t F(x)$ for all $x \in H$,

(b) (subadditivity) $W(F+G) \subset W(F)+W(G)$ for all $F, G \in M(H)$, with $F+G \in M(H)$ being defined by $(F+G)(x)=F(x)+G(x)$ for all $x \in H$,

(c) (traslation property) $W(\alpha I+F)=\alpha+W(F)$ for all $\alpha \in \mathbb{R}$, for all $F \in M(H)$, with $I: H \rightarrow H$ denoting the identity operator,

(d) (inner-semicontinuity) $W$ (innlim $\left.F_{k}\right) \subset$ innlim $W\left(F_{k}\right)$ for any sequence $\left\{F_{k}\right\}_{k \in \mathbb{N}}$ in $M(H)$,

(e) (spectral containment) $\sigma(F) \subset W(F)$ for all $F \in M(H)$.

Our discussion on the inner-semicontinuity of $\sigma$ is centered around the spectral containment property. By working out a few simple examples, one quickly realizes that in most cases the spectral containment property holds in a strict form. In other words, one should not expect the reverse inclusion $W(F) \subset \sigma(F)$ to occur, unless $F$ has a very special structure. The next proposition describes a peculiar type of operator $F$ for which $W(F)$ does coincide with $\sigma(F)$. The notation $\Pi_{K}: H \rightarrow H$ refers to the metric projection onto the closed convex set $K \subset H$, i.e. $\Pi_{K}(y)$ is defined as the unique solution to the minimization problem

$$
\text { Minimize }\{\|y-u\|: u \in K\} \text {. }
$$

Proposition 5.3. Let $F: H \rightrightarrows H$ be innerward in the sense that

$$
\Pi_{\mathbb{R} x}(F(x)) \subset F(x) \quad \text { for all } x \in H \backslash\{0\} .
$$

Then, $W(F)=\sigma(F)$.

Proof. For $x \in H \backslash\{0\}$, the metric projection of $y \in H$ onto the closed subspace $\mathbb{R} x:=\{\alpha x: \alpha \in \mathbb{R}\}$ is given explicitly by

$$
\Pi_{\mathbb{R} x}(y)=\frac{\langle x, y\rangle}{\langle x, x\rangle} x .
$$

The innerwardness of $F$ amounts to saying that

$$
\frac{\langle x, y\rangle}{\langle x, x\rangle} x \in F(x) \quad \text { for all } x \neq 0 \text { and all }(x, y) \in \operatorname{Gr} F \text {. }
$$

In particular,

$$
\frac{\langle x, y\rangle}{\langle x, x\rangle} \in \sigma(F) \quad \text { for all } x \neq 0 \text { and all }(x, y) \in \operatorname{Gr} F,
$$

showing in this way the remaining inclusion $W(F) \subset \sigma(F)$. 
REMARK. The $F_{k}^{\prime} s$ introduced in Example 1.3 are all of them innerward. As we shall see in a moment, this fact explains why $\sigma$ behaves inner-semicontinously with respect to the sequence $\left\{F_{k}\right\}_{k \in \mathbb{N}}$.

Since the equality $W(F)=\sigma(F)$ is in general difficult to obtain, it seems reasonable to ask whether the inclusion

$$
W(F) \subset \mathcal{H}(\sigma(F))
$$

holds for a suitable enlargement mapping $\mathcal{H}: P(\mathbb{R}) \rightarrow P(\mathbb{R})$. The term "enlargement" attributed to $\mathcal{H}$ refers to the property

$$
S \subset \mathcal{H}(S) \text { for all } S \in P(\mathbb{R}) \text {. }
$$

Two prototypes of enlargement mappings that we have in mind are

$$
\begin{array}{ll}
\mathcal{H}(S)=\operatorname{cl} S & \text { (topological closure) } \\
\mathcal{H}(S)=\operatorname{co} S & \text { (convex hull) }
\end{array}
$$

but one may consider also other more sophisticate examples. Without further ado we state:

Lemma 5.4. Let $\mathcal{H}: P(\mathbb{R}) \rightarrow P(\mathbb{R})$ be an arbitrary enlargement mapping. Let $\left\{F_{k}\right\}_{k \in \mathbb{N}} \subset M(H)$ be a sequence such that

$$
W\left(F_{k}\right) \subset \mathcal{H}\left(\sigma\left(F_{k}\right)\right) \quad \text { for all } k \in \mathbb{N} .
$$

Then $\sigma\left(\operatorname{innlim} F_{k}\right) \subset \operatorname{innlim} \mathcal{H}\left(\sigma\left(F_{k}\right)\right)$.

Proof. With Proposition 5.2 at hand, it is easy to see that

$$
\sigma\left(\operatorname{innlim} F_{k}\right) \subset W\left(\operatorname{innlim} F_{k}\right) \subset \operatorname{innlim} W\left(F_{k}\right) \subset \operatorname{innlim} \mathcal{H}\left(\sigma\left(F_{k}\right)\right) .
$$

Without invoking Proposition 5.2, one may proceed as follows: let $\lambda \in \mathbb{R}$ be in the point spectrum of innlim $F_{k}$. Then, there is a nonzero vector $x$ such that $(x, \lambda x) \in \operatorname{innlim} \operatorname{Gr} F_{k}$. As a consequence, one can write

$$
(x, \lambda x)=\lim \left(x^{k}, y^{k}\right) \quad \text { with }\left(x^{k}, y^{k}\right) \in \operatorname{Gr} F_{k} \quad \text { for all } k \in \mathbb{N} .
$$

Since $x \neq 0$, one may suppose that $x^{k} \neq 0$ for all $k \in \mathbb{N}$. Hence,

$$
\frac{\left\langle x^{k}, y^{k}\right\rangle}{\left\langle x^{k}, x^{k}\right\rangle} \in W\left(F_{k}\right) \subset \mathcal{H}\left(\sigma\left(F_{k}\right)\right) .
$$

Observing that

$$
\frac{\left\langle x^{k}, y^{k}\right\rangle}{\left\langle x^{k}, x^{k}\right\rangle} \rightarrow \frac{\langle x, \lambda x\rangle}{\langle x, x\rangle}=\lambda,
$$

one arrives at $\lambda \in \operatorname{innlim} \mathcal{H}\left(\sigma\left(F_{k}\right)\right)$. 
Theorem 5.5. Suppose that for some enlargement mapping $\mathcal{H}: P(\mathbb{R}) \rightarrow$ $P(\mathbb{R})$, the sequence $\left\{F_{k}\right\}_{k \in \mathbb{N}}$ satisfies the following two conditions:

(a) $W\left(F_{k}\right) \subset \mathcal{H}\left(\sigma\left(F_{k}\right)\right)$ for all $k \in \mathbb{N}$,

(b) $\left\{\sigma\left(F_{k}\right)\right\}_{k \in \mathbb{N}}$ and $\left\{\mathcal{H}\left(\sigma\left(F_{k}\right)\right)\right\}_{k \in \mathbb{N}}$ have the same inner-limit.

Then $\sigma\left(\operatorname{innlim} F_{k}\right) \subset \operatorname{innlim} \sigma\left(F_{k}\right)$.

Proof. It is an immediate consequence of Lemma 5.4.

Corollary 5.6. Let $\left\{F_{k}\right\}_{k \in \mathbb{N}}$ be a sequence such that $W\left(F_{k}\right) \subset \operatorname{cl}\left[\sigma\left(F_{k}\right)\right]$ for all $k \in \mathbb{N}$. Then,

$$
\sigma\left(\operatorname{innlim} F_{k}\right) \subset \operatorname{innlim} \sigma\left(F_{k}\right) .
$$

Proof. It suffices to apply Theorem 5.5 with the choice $\mathcal{H}=\mathrm{cl}$. The assumption (b) is automatically satisfied because the concept of inner-limit is blind to the closure operation on sets.

\section{REFERENCES}

[1] R. Arens, Operational calculus of linear relations, Pacific J. Math. 11 (1961), 9-23.

[2] J. B. Conway And B. B. Morrel, Operators that are points of spectral continuity, Integral Equations Operator Theory 2 (1979), 174-198.

[3] P. Halmos, A Hilbert Space Problem Book (2nd Edition), Springer-Verlag, New-York, 1982.

[4] A. D. Ioffe, Nonsmooth analysis: differential calculus of nondifferentiable mappings, Trans. Amer. Math. Soc. 266 (1981), 1-56.

[5] J. D. Newburgh, The variation of spectra, Duke Math. J. 18 (1951), 165-176.

[6] R. T. Rockafellar, Convex Analysis, Princeton Univ. Press, Princeton, N. J., 1970.

[7] A. Seeger, Spectral analysis of set-valued mappings, Acta Math. Vietnam. 23 (1998), 49-63.

[8]_Eigenvalue analysis of equilibrium processes defined by linear complementarity conditions, Linear Algebra Appl. 292 (1999), 1-14.

Manuscript received September 30, 1999

Philippe Lavilledieu and Alberto Seeger

Department of Mathematics

University of Avignon

33, rue Louis Pasteur

84000 Avignon, FRANCE

E-mail address: Philippe.Lavilledieu@univ-avignon.fr, Alberto.Seeger@univ-avignon.fr 